\title{
1 Adding a further twist to the tail of Leptospirosis in the UK
}

2 Sally J. Cutler

3 School of Health, Sports \& Bioscience, University of East London, London, E15 4LZ.

4

5 Conventional serological typing of the spirochaete Leptospira (figure 1) is

6 challenging, particularly when applied to serogroup Pomona. This group being

7 comprised of members of four genospecies, namely Leptospira interogans

8 (Kennewicki; Monjakov; Pomona), Leptospira kirschneri (Altodouro; Mazdok;

9 Tsaratsova; Kumming), Leptospira noguchii and Leptospira sanarosai. The latter two species not being endemic to Europe. The significance attributed to these strains is hugely variable with L. kirschneri seorvar Mozdock, only rarely resulting in consequences amongst livestock or companion animals, whereas $L$. interrogans serovars Pomona type Kennewicki potentially results in devastating infection consequences (Timoney and others 2011). The pathogenic traits of Kennewicki strains are not shared with other serovars belonging to the L. interogans Pomona serogroup such as Monjakov or Pomona. In Europe, within the Pomona serogroup, serovars Pomona and Mozdok correlated pigs (figure 2) and rodent reservoirs respectively are the most commonly encountered members of this group, with occasional spill-over into non-reservoir species. Expansion of reservoir species has been reported for serovar Pomona with its ability infect sea lions, but also cause disease (Prager and others 2013).

Leptospiral infection associated with serogroup Pomona has been associated with haemorrhagic acute febrile manifestations, renal signs, jaundice, and reproductive involvement (Jacobs and others 2015). Equines appear to be particularly susceptible 
with several reports of abortion, particularly where serovar Kennewicki is endemic

27 (Timoney and others 2011). Intriguingly, the majority of isolates assessed by Arent et al, are largely derived from equine infection (symptomatic and asymptomatic) over a three-year period (Arent and others 2017a).

30

Given this backdrop, the description of serogroup Pomona from the UK domestic animals was concerning. This serogroup has been sporadically reported from livestock in the UK, with both Mozdock and Pomona serovars being recovered. Arent and co-workers (this issue), subjected a series of 10 UK-derived isolates to various Leptospiral typing approaches to gain insights into their identity and enable assessment of their potential pathogenic potential. Recovery of isolates is technically challenging, hence our general reliance upon non-cultivation based diagnostics such as serology and molecular detection. Evolution of molecular typing techniques has enabled more highly discriminatory methods to be applied to the Leptospiral group and has highlighted the heterogeneity even within serovars and facilitated analysis of these sub-populations by host and geographical location (Arent and others 2017b) and has been used to describe new strains such as Altodouro (Paiva-Cardoso and others 2013).

44

Application of molecular typing revealed that the isolates all resembled serovar Pomona, a finding that supports the greater potential of this serovar to spill into livestock species. Interestingly, restriction endonuclease digestion using $A / u l$ and Hpall could discriminate between two sub-populations amongst the recovered isolates, splitting those recovered from animals in Northern Ireland and that obtained 
51 England. This sub-division could not be resolved by MLVA raising the question of the

52 discriminatory capability of these two typing methods? This conundrum is akin to that

53 which these authors previously assessed with different serovars of $L$. interrogans

54 Bratislava and Muenchen, where again restriction endonuclease digestion offered

55 greater resolution (Arent and others 2016). These data raise the question as to

56 whether restriction endonuclease digestion should be retained as a valued highly

57 discriminatory tool over methods such as MLVA which offers greater transportability

58 of data between laboratories, and requires significantly less DNA as a pre-requisite

59 for typing? Under stringently controlled conditions, restriction endonuclease digestion

60 appears to retain its value for discrimination of sub-types within serotype, but this

61 could also suggest that alternative MLVA approaches need to be further refined with

62 a view of increasing their discriminatory power. It maybe that an alternative typing

63 approach such as use of canonical SNPs might provide a more transferrable and

64 less DNA thirsty highly discriminatory solution for molecular typing of Leptospira?

65

66 To conclude, the isolates recovered from sporadic testing in the UK revealed that

67 Pomona was the causative serovar, thus paralleling the observations seen elsewhere in Europe where Pomona serogroup strains infect livestock. Interestingly,

69 a new variant was described. As this currently was based upon a single isolate from

70 a shrew, further investigative studies are essential to map strain epidemiology and

71 assess host correlations and their pathogenic potential. 
74 References:

ARENT, Z., FRIZZELL, C., GILMORE, C., ALLEN, A. \& ELLIS, W. A. (2016)

Leptospira interrogans serovars Bratislava and Muenchen animal infections:

77

Implications for epidemiology and control. Veterinary Microbiology 190, 19-26

ARENT, Z., GILMORE, C., BARLOW, A. M., SMITH, L. \& ELLIS, W. A. (2017a)

79

Leptospira interrogans serogroup Pomona infections in the UK: is there a real threat for farm animals? Veterinary Record

ARENT, Z. J., GILMORE, C., SAN-MIGUEL AYANZ, J. M., NEYRA, L. Q. \&

GARCÍA-PEÑA, F. J. (2017b) Molecular Epidemiology of Leptospira Serogroup

Pomona Infections Among Wild and Domestic Animals in Spain. EcoHealth 14, 48-

$84 \quad 57$

JACOBS, A. A. C., HARKS, F., HOEIJMAKERS, M., COLLELL, M. \& SEGERS, R.

P. A. M. (2015) Safety and efficacy of a new octavalent combined Erysipelas, Parvo and Leptospira vaccine in gilts against Leptospira interrogans serovar Pomona associated disease and foetal death. Vaccine 33, 3963-3969

PAIVA-CARDOSO, M. D.

D. N., ARENT, Z., GILMORE,

C., HARTSKEERL, R. \&

ELLIS, W. A. (2013) Altodouro, a new Leptospira serovar of the Pomona serogroup isolated from rodents in northern Portugal. Infection, Genetics and Evolution 13, 211217

PRAGER, K. C., GREIG, D. J., ALT, D. P., GALLOWAY, R. L., HORNSBY, R. L.,

PALMER, L. J., SOPER, J., WU, Q., ZUERNER, R. L., GULLAND, F. M. D. \& LLOYD-SMITH, J. O. (2013) Asymptomatic and chronic carriage of Leptospira interrogans serovar Pomona in California sea lions (Zalophus californianus).

97 Veterinary Microbiology 164, 177-183 
98 TIMONEY, J. F., KALIMUTHUSAMY, N., VELINENI, S., DONAHUE, J. M.,

99 ARTIUSHIN, S. C. \& FETTINGER, M. (2011) A unique genotype of Leptospira

100 interrogans serovar Pomona type kennewicki is associated with equine abortion.

101 Veterinary Microbiology 150, 349-353

102 